\title{
Remote sensing of the Io torus plasma ribbon using natural radio occultation of the Jovian radio emissions
}

\author{
M. Y. Boudjada ${ }^{1}$, P. H. M. Galopeau ${ }^{2}$, S. Sawas ${ }^{3}$, and H. Lammer ${ }^{1}$ \\ ${ }^{1}$ Space Research Institute, Austrian Academy of Sciences, Graz, Austria \\ ${ }^{2}$ Université Versailles St-Quentin, CNRS/INSU, LATMOS-IPSL, Guyancourt, France \\ ${ }^{3}$ Institute of communications and wave propagation, University of Technology, Graz, Austria \\ Correspondence to: M. Y. Boudjada (mohammed.boudjada@ oeaw.ac.at)
}

Received: 20 December 2013 - Revised: 21 May 2014 - Accepted: 27 June 2014 - Published: 9 September 2014

\begin{abstract}
We study the Jovian hectometric (HOM) emissions recorded by the RPWS (Radio and Plasma Wave Science) experiment onboard the Cassini spacecraft during its Jupiter flyby. We analyze the attenuation band associated with the intensity extinction of HOM radiation. This phenomenon is interpreted as a refraction effect of the Jovian hectometric emission inside the Io plasma torus. This attenuation band was regularly observed during periods of more than 5 months, from the beginning of October 2000 to the end of March 2001. We estimate for this period the variation of the electron density versus the central meridian longitude (CML). We find a clear local time dependence. Hence the electron density was not higher than $5.0 \times 10^{4} \mathrm{~cm}^{-3}$ during 2 months, when the spacecraft approached the planet on the dayside. In the late afternoon and evening sectors, the electron density increases to $1.5 \times 10^{5} \mathrm{~cm}^{-3}$ and reach a higher value at some specific occasions. Additionally, we show that ultraviolet and hectometric wavelength observations have common features related to the morphology of the Io plasma torus. The maxima of enhancements/attenuations of UV/HOM observations occur close to the longitudes of the tip of the magnetic dipole in the southern hemisphere $\left(20^{\circ} \mathrm{CML}\right)$ and in the northern hemisphere $\left(200^{\circ} \mathrm{CML}\right)$, respectively. This is a significant indication about the importance of the Jovian magnetic field as a physical parameter in the coupling process between Jupiter and the Io satellite.
\end{abstract}

Keywords. Magnetospheric physics (planetary magnetospheres)

\section{Introduction}

\subsection{Io plasma torus}

Io is the innermost of the four large Galilean satellites of Jupiter and the most volcanically active body in the solar system. This volcanic activity provides Io with a fluctuating $\mathrm{SO}_{2}$ atmosphere where important fractions are lost in the form of neutral oxygen and sulfur atoms. These materials form extended clouds along Io's orbit, and over time are ionized by two main processes: electron impact and charge exchange reactions. The ionized components are rapidly accelerated under the effect of the Jovian magnetic field. These ions form a ring-like structure known as Io plasma torus whose electron density is about $2000 \mathrm{~cm}^{-3}$ (Steffl et al., 2004a).

\subsection{Plasma and UV observations of the Io torus}

Over the past 30 years the Io plasma torus has been observed remotely from ground-based stations and from experiments onboard spacecraft. Prior to the Voyager mission, groundbased optical observations of the region near Io and its orbit have revealed the presence of neutral sodium and ionized sulfur (Kupo et al., 1976). Those first observations certainly mark the discovery of the plasma torus at Io which has been confirmed by Voyager 1 when it approached Jupiter to a periapsis distance of $4.89 R_{\mathrm{J}}$. The observations of the Voyager1 plasma science instrument found three components in the Io torus: the cold inner torus, a "ribbon" just inside the Io's orbit, and the warm outer torus. The cold $(<1 \mathrm{eV})$ and hot $(\sim 30 \mathrm{eV})$ parts of the torus have electron density peaks of about $10^{3} \mathrm{~cm}^{-3}$ and $2 \times 10^{3} \mathrm{~cm}^{-3}$ at $\sim 5.3$ and $\sim 6 R_{\mathrm{J}}$, respectively. At a radial location which varies between 5.6 
and $5.9 R_{\mathrm{J}}$, the ribbon has peak densities on the order of $3 \times 10^{3} \mathrm{~cm}^{-3}$ (Bagenal and Sullivan, 1981).

The measurements of electron density were performed by the plasma wave subsystem instruments on Galileo spacecraft during its pass through the torus on December 1995. The Galileo observations have found different results from those of Voyager 1; in particular, between 7.8 and $5.9 R_{\mathrm{J}}$, Galileo observed density enhancement by approximately a factor 2 (Gurnett et al., 1996). Shortly after crossing Io's orbit, the density profile dropped sharply and remained at low values suggesting that the "ribbon" region was either absent or much farther from Jupiter than usual (Bagenal et al., 1997).

Krüger et al. (2003) reported an increase by 2-3 times in dust emission rate from Io during Galileo's G28 orbit from July to December 2000. Similar short-term (e.g., few months) variations have been reported by Brown and Bouchez (1997), who found an increase of $30 \%$ in torus mass. The origins of these variations were attributed to changes in Io's volcanic activity. A detailed analysis of such short-term variations associated with the Io torus was performed by the Ultraviolet Imaging Spectrograph, UVIS (Esposito et al., 2004), onboard the Cassini spacecraft, from October 2000 to March 2001. The broad spectral range, high spectral resolution (compared to Voyager), and temporal coverage of the UVIS experiment resulted in the creation of a unique and rich data set of the Io plasma torus in the ultraviolet.

In a series of four papers Steffl et al. (2004a, b, 2006 and 2008) analyzed the temporal, radial and azimuthal variations of the Io plasma torus during the Cassini encounter with Jupiter. The observed radial profile of the electron column density was well matched by assuming that the local electron number density profile was proportional to $r^{-5.4}$ from 6.0 to $7.8 R_{\mathrm{J}}$ and $r^{-12}$ outside of $7.8 R_{\mathrm{J}}$. The slope of the electron density derived from UV spectra was found to be almost identical to the slope of the model of Bagenal (1994) based on Voyager observations. Steffl et al. (2006) analyzed the relative variations of electron density and electron temperature with CML (central meridian longitude). The average variation in electron density, observed in the period from 10 October to 14 November 2000, was found anti-correlated with the average variation in electron temperature. The electron density reached a maximum value near $160^{\circ} \mathrm{CML}$, while the electron temperature has a minimum value near $170^{\circ} \mathrm{CML}$.

\subsection{Attenuation of Jovian hectometric emissions by the Io plasma torus}

Radio emissions from Jupiter have been observed on Earth for nearly 6 decades, since the discovery of the Jovian decametric (DAM) radiation (10-40 MHz) by Burke and Franklin (1955). Space observations have provided, at lower frequencies (less than $5 \mathrm{MHz}$ ), the possibility of avoiding the ionospheric opacity and detecting the Jovian hectometric emission observed in the frequency range between $300 \mathrm{kHz}$ and $3 \mathrm{MHz}$ (Kaiser, 1977). The Voyager mission to the giant planets provided further detailed information on the spectral properties of the Jovian radio emissions, in particular the hectometric (HOM) wavelength emissions. The Planetary Radio Astronomy (PRA) experiment onboard Voyager spacecraft detected Jovian emissions in its low-frequency band, which extends from 1.2 to $1326 \mathrm{kHz}$. HOM was found to be strongly modulated by the rotation of the planet, with peaks of about $100 \%$ occurrence probability near 100 and $330^{\circ}$ in CML. This radiation is mostly observed at frequencies between about 500 and $1300 \mathrm{kHz}$ with excursions both above and below these limits (Barrow and Desch, 1989). Further investigations based on the Galileo mission to Jupiter showed that the upper frequency of HOM may be as high as $5.6 \mathrm{MHz}$ (Galopeau and Boudjada, 2005). The polarization of HOM emissions was widely investigated by OrtegaMolina and Lecacheux (1991). Their findings strongly indicated that this emission is predominantly right-hand polarized when observed from the Jovian northern hemisphere and left-hand polarized when observed from the southern hemisphere. The polarization measurements suggested that the HOM emission is generated by the cyclotron maser instability (Wu and Lee, 1979) in the R-X mode from high-latitude source regions in both hemispheres (Galopeau et al., 2004).

On the basis of the Voyager observations, Warwick et al. (1979) were able to construct a plasma density contour map of the Io plasma torus. The Voyager 1 approach provided direct in situ measurements within a Jovicentric distance of $4.9 R_{\mathrm{J}}$. The authors determined the electron densities from the radio data (PRA experiment) obtained on both inbound and outbound legs of the spacecraft trajectory on 5 March 1979, between 06:00 and 16:00 SET (spacecraft event time). The Voyager PRE observations recorded a distinct narrow band of emission at the local electron upper hybrid frequency $f_{\mathrm{uh}}=\sqrt{f_{\mathrm{p}}^{2}+f_{\mathrm{c}}^{2}}$, where the cyclotron frequency, $f_{\mathrm{c}}(\mathrm{MHz})=2.8 \mathrm{~B}$ (Gauss), can be estimated from the local magnetic field and the plasma frequency, $f_{\mathrm{p}}=9 \sqrt{N_{\mathrm{e}}}$, allows determining the local density, $N_{\mathrm{e}}$ (in $\mathrm{cm}^{-3}$ ). The spacecraft traversed the outer torus in the mid-afternoon inbound and late evening outbound. Voyager 1 moved through the inner torus near closest approach. This spacecraft trajectory gave the opportunity to draw contours of constant density in a meridional plane by assuming the torus to be symmetric both azimuthally and about the magnetic equator. Hence the authors constructed a plasma density contour map of the Io plasma torus whose $500 \mathrm{~cm}^{-3}$ contour extended from inside $5 R_{\mathrm{J}}$ to nearly $8 R_{\mathrm{J}}$ in the equatorial plane and over $1 R_{\mathrm{J}}$ above and below the plane. Extrapolations suggested a maximum electron density in the plane of above $3500 \mathrm{~cm}^{-3}$ near $5.6 R_{\mathrm{J}}$.

The PRA experiment onboard the Voyager 1 spacecraft revealed the presence of "drifting gaps" in the Jovian hectometric radio emission spectrum. Lecacheux (1981) attempted to 
explain the origin of such HOM spectral features. The author simulated the radio wave propagation in the vicinity of the Io plasma torus. He showed that the origin of the "drifting gaps" was due to refraction of HOM emissions in the Io torus plasma. Additionally, another model has been suggested by Gurnett et al. (1998) where the extinction of the HOM emission is caused by coherent scattering from short wavelength density fluctuations or shallow-angle reflection from field-aligned density irregularities near the Io $\mathrm{L}$ shell. A ray tracing study carried out by Menietti et al. (2003) investigated the source location of the attenuation lanes. The combination of Galileo and Cassini observations at numerous frequencies allowed the authors to find that the attenuation occurred at subauroral regions along a magnetic field flux tube which interacts with Io. The location of the attenuation as derived by the ray-tracing technique gives support to the model suggested by Gurnett et al. (1998). Boudjada et al. (2011) investigated the attenuation band recorded by the Cassini/RPWS (Radio and Plasma Wave Science) experiment from 20 November 2000 to 13 January 2001. The authors showed regular and systematic observations of the attenuation band suggesting that the plasma medium at the origin of these features is steady and stable. The Io torus may be considered as the most probable plasma medium where the HOM emission is refracted through its ray path propagation. Boudjada et al. (2011) suggested that the Lecacheux model is more adapted to explain the attenuation frequency band.

Other contributions characterized the spectral features of HOM "drifting gaps" (also called attenuation lanes, attenuation bands, and lanes of decreased emission) using the Voyager data (Green et al., 1992; Higgins et al., 1995, 1998) and also further missions to Jupiter like Galileo (Gurnett et al., 1998; Boudjada et al., 2001; Menietti et al., 2001, 2003) and Cassini (Menietti et al., 2003; Boudjada et al., 2011). Those investigations found the following attenuation band characteristics:

a. morphological aspects which showed that the lanes are at least semi-permanent features of HOM emission (Higgins et al., 1995),

b. its relationship to the Io volcanic activity (Menietti et al., 2001) and

c. the regular and systematic observations of this phenomenon suggest that the plasma medium at the origin of the HOM extinction is steady and stable (Boudjada et al., 2011).

The aim of this paper is to investigate the refraction of the Jovian hectometric emissions by the Io torus leading to deriving the electron density in the Io plasma torus during the Cassini's Jupiter flyby. In the next section we describe the method we use to estimate the attenuation frequency from the dynamic spectra recorded by the Cassini/RPWS experiment. In Sect. 3 we select three main intervals of the Cassini trajectory taking into consideration the local time (LT) and the

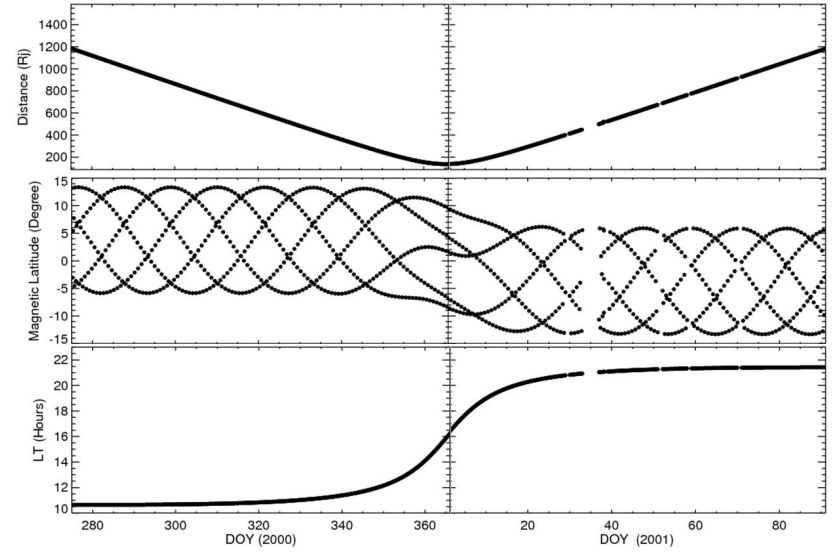

Figure 1. Variation of Cassini orbital parameters versus the DOY during the Jupiter flyby. The investigated period is from 1 October 2000 (DOY 265) to 31 March 2001 (DOY 090), and the closest approach to the planet was on 27 December 2001. The distance between Jupiter and the spacecraft (expressed in Jovian radii), the magnetic latitude (in degrees) and local time (in hours) are shown, respectively, in the top, middle and bottom panels.

distance to the planet. We derive the corresponding electron density versus LT time intervals. We discuss in Sect. 4 our results and compare them to previous radio and UV observations recorded from ground-based stations and spacecraft. The main outcomes are summarized in Sect. 5 and future perspectives are outlined.

\section{Data analysis}

The data used in this paper were obtained by the Cassini spacecraft's RPWS experiment (Gurnett et al., 2004) during its Jupiter flyby between 1 October 2000 and 31 March 2001. The RPWS experiment is designed to measure the electric and magnetic fields of radio emissions and plasma waves across a broad range of frequencies. The electric field measurements are recorded by three nearly orthogonal antennas over a frequency range from $1 \mathrm{~Hz}$ to $16 \mathrm{MHz}$. We focus on the RPWS observations recorded by the high-frequency receiver (hereafter HFR) in the range between $100 \mathrm{kHz}$ and 16.125 MHz. We concentrate on the intensity extinction of the Jovian HOM emission which occurs, on average, between $300 \mathrm{kHz}$ and $5 \mathrm{MHz}$.

\subsection{Cassini flyby of Jupiter}

Figure 1 summarizes the main orbital parameters of the Cassini spacecraft before, during, and after the close approach to Jupiter. The two upper panels show the variation of the distance (expressed in Jovian radii $R_{\mathrm{J}}$ ) and the satellite magnetic latitude versus the day of the year - DOY. In the beginning of October 2000, the spacecraft moved towards the southern hemisphere of the planet with variable magnetic 

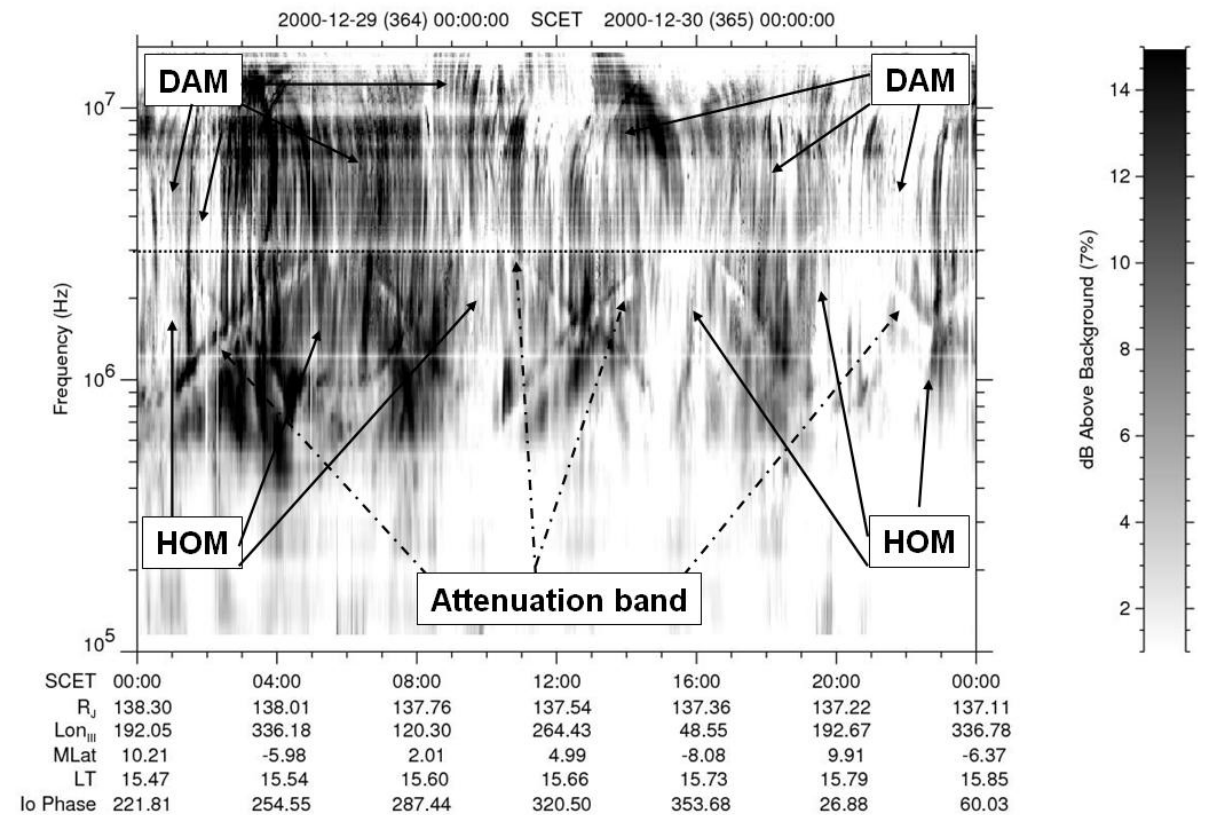

Figure 2. Dynamic spectrum recorded by the Cassini RPWS experiment on 29 December 2000. The Jovian DAM and HOM emissions principally occur in the frequency ranges of $16-8 \mathrm{MHz}$ and $3000-100 \mathrm{kHz}$, respectively. The horizontal dashed line (at $3 \mathrm{MHz}$ ) indicates the common way to recognize the upper frequency of Jovian HOM emissions. The attenuation band appears as incomplete sinusoid traces occurring when the northern and southern magnetic field axes are toward the observer (i.e., Cassini spacecraft). Both traces cross each other in the equatorial plane of the planet (e.g., 03:30, 08:00, 12:45, 18:00 and 23:30 UT).

latitude between -6 and $+13^{\circ}$ at a distance of about $1200 R_{\mathrm{J}}$. The closest approach was on 30 December 2000 at a distance of $137 R_{\mathrm{J}}$ and magnetic latitude from -10 to $+9^{\circ}$. In the end of March 2001, the Cassini spacecraft was at a distance of more than $1200 R_{\mathrm{J}}$ with magnetic latitude between +6 and $-14^{\circ}$. The LT variation of the spacecraft versus the DOY is displayed in the bottom panel of Fig. 1. In the investigated period the spacecraft approached and left, respectively, the morning/noon (i.e., 10:40-13:00 LT) and late evening (i.e., 19:30-21:30 LT) sectors of the planet. During the closest approach the spacecraft LT range changed in 10 days from about 13:30 to 19:00 LT. Gaps in the data occurred at the beginning of February (from 1 to 4 ) and also partial gaps of more than $12 \mathrm{~h}$ took place at the end of the year $2000(20,21$, 22, 25, and 26 December) and on 28 February 2001.

\subsection{Overview of the attenuation band}

The Jovian radio emissions were regularly and daily recorded. The observations were displayed in the dynamic spectrum, i.e., intensity variation versus the frequency and the time. Figure 2 shows a typical dynamic spectrum recorded 1 day before the closest approach to the planet. The Jovian hectometric emission principally occurred in the frequency range from $100 \mathrm{kHz}$ to about $3 \mathrm{MHz}$. HOM emissions are modulated by the planetary rotations (of about $10 \mathrm{~h}$ ) which occur in the time intervals 02:30-12:30 UT and 12:3022:30 UT. Typical attenuation bands are seen in Fig. 2. We note an enhancement of the edges of the attenuation band observed at three time intervals 01:00-05:00, 06:30-09:00 and 10:00-14:00 UT. The outline of the attenuation band on the dynamic spectrum appears as an incomplete sinusoid.

During a full Jovian rotation one observes a succession of two incomplete sinusoids associated with the northern and southern hemispheres. These sinusoids cross each other at about 03:30, 08:00, 12:45, 18:00 and 23:30 UT as displayed in Fig. 2. These intersections correspond to periods when the spacecraft is in the Jovian magnetic equator plane, i.e., the spacecraft magnitude latitude is almost equal to zero. According to Lecacheux model (1981) we alternatively observe the extinction by the Io torus in the Jovian hectometric emission emitted from the southern or northern hemisphere, respectively, when the magnetic latitude has a negative or a positive value. The main phenomenological aspects of the attenuation bands, as observed by the Cassini/RPWS experiment, have been described in Boudjada et al. (2011).

\subsection{The selected LT time interval}

The attenuation band was continuously detected during more than 5 months, from the beginning of October 2000 to the end of March 2001, corresponding to more than 400 Jovian rotations. The intensity levels of the center and the edge parts of the HOM attenuation band are found to be, on average, of about 3 and $15 \mathrm{~dB}$, respectively, as can be seen in Fig. 2. We derive from the dynamic spectra the observational 
parameters where the extinction of the Jovian hectometric emission occurs: the observation time, the center frequency (called hereafter attenuation band frequency) and the central meridian longitude. First, we use a cursor procedure to select and to save the observation time, the frequency and the intensity level of the attenuation band. Second, we derive the central meridian longitude (from the spacecraft orbital positions with regard to Jupiter) related to the observation time. This leads us to study the variation of the attenuation band frequency versus the CML, as shown in Fig. 3 . We note that the HOM intensity extinction occurred, on average, in the frequency range between $500 \mathrm{kHz}$ and $3.5 \mathrm{MHz}$. However we also observe that the attenuation of HOM emissions can reach frequencies of up to $8 / 10 \mathrm{MHz}$ which corresponds to less than $1 \%$ of the total points considered in this study. Another important characteristic is the clear overlapping, at the equatorial plane, of the extinction of HOM emissions coming from the southern and northern hemispheres, as shown in Fig. 2. The overlapping is observed at two CML interval longitudes, between $80-110^{\circ}$ and $270-300^{\circ}$ CMLs. In the first interval we observe that the frequency of the HOM intensity extinction coming from the southern/northern hemisphere is decreasing/increasing versus the central meridian longitude. In the second longitude interval we find that the frequency of the HOM attenuation band coming from the southern/northern hemisphere is increasing/decreasing against the CML. We distinguish in Fig. 2 between both hemispheres by using the criteria suggested by Ortega-Molina and Lecacheux (1991). HOM radiation emitted by the northern hemisphere is mainly right-hand circular polarized and occurred in the CML range between 60 and $330^{\circ}$, and the southern hemisphere is mainly left-hand circular polarized and occurred in the CML ranges $330-360^{\circ}$ and $0-60^{\circ}$. The previous investigation was based on the sense of radio wave polarization (right- and left-hand circular polarizations) as recorded by the PRA experiment onboard Voyager spacecraft.

\subsection{Method of data investigation}

An important feature of the HOM intensity extinction is its repeatability. The hectometric attenuation frequency can be modeled by two incomplete sinusoids; each one is due to the extinction of HOM emission coming from one hemisphere. After we proceed to the estimation of the electron density in the Io torus, we suppose that the refraction occurs when the plasma frequency of the Io torus is equal to the attenuated frequency of the Jovian hectometric emission. This leads us to derive the electron density using the well-known relationship

$f_{\mathrm{p}}=9 \sqrt{N_{\mathrm{e}}}$,

where $f_{\mathrm{p}}$ and $N_{\mathrm{e}}$ are the plasma frequency (in $\mathrm{kHz}$ ) and the electron density $\left(\mathrm{in}^{-3}\right.$ ).

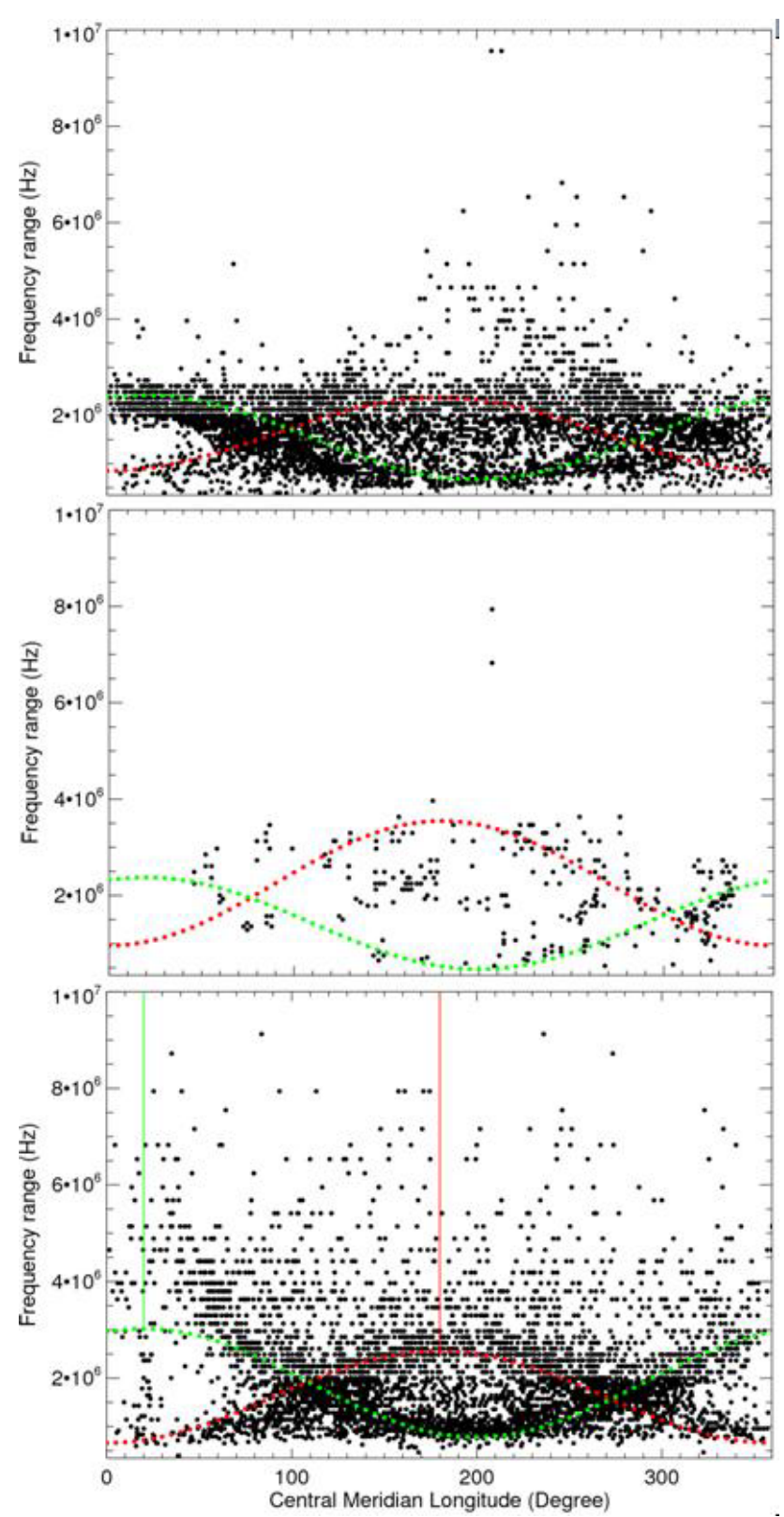

Figure 3. Attenuation band frequency $(\mathrm{Hz})$ versus the CML. The top, middle and bottom panels correspond, respectively, to the three LT time intervals: 10:40-13:00 LT, 13:30-19:00 LT, and 19:3021:30 LT. The red and green sinusoidal curves correspond to the fitting of the attenuation frequency bands related, respectively, to the northern and southern hemispheres. The two maximum frequencies at $20^{\circ} \mathrm{CML}$ (green vertical line) and $180^{\circ} \mathrm{CML}$ (red vertical line) shown in the bottom panel are connected, respectively, to the time when the tip of the Jovian magnetic axis, in the northern or southern hemisphere, is tilted toward the observer. 


\section{Electron density as inferred from HOM refraction effect}

Figure 3 shows the variation of the frequency of the HOM intensity extinction versus the central meridian longitude for the three selected LT intervals. This separation leads us to characterize how the HOM emission is attenuated taking into consideration the geometric configurations of Cassini spacecraft with regards to the Sun and to Jupiter. Three regions of the Jovian magnetosphere - the morning, the afternoon and the evening sectors - are covered. The corresponding attenuation bands of the HOM emissions are displayed in the three panels of Fig. 3 corresponding to the selected intervals 10:40-13:00 LT (top panel), 13:30-19:00 LT (middle panel), and 19:30-21:30 (bottom panel). After this LT division, we proceed to the fitting of the attenuation frequency band versus the central meridian longitude by sinusoid curves. The red and green sinusoidal modulations in Fig. 3 correspond to the fitting of the frequency attenuation bands related, respectively, to the northern and southern hemispheres.

\subsection{Late morning and early afternoon sectors}

The HOM intensity extinction as shown in the top panel of Fig. 3 was recorded from 1 October to 20 December 2000. During this period the distance of the spacecraft decreases respectively from 1180 to $188 R_{\mathrm{J}}$, when the local time changes from 10:40 to 13:00 LT. Of the total amounts of points, $47 \%$ have been observed in this LT interval. The HOM attenuation occurs, on average, between $500 \mathrm{kHz}$ and $3 \mathrm{MHz}$. The particular feature of this time interval is the quasi-systematic "cutoff" of the HOM emission at about $3 \mathrm{MHz}$. From the sinusoidal curves we find two maximum attenuation frequencies on the order of $2400 \mathrm{kHz}$ have been observed at 20 and $180^{\circ} \mathrm{CML}$, and two minima of about 850 and $700 \mathrm{kHz}$ have been recorded at 0 and $200^{\circ} \mathrm{CML}$. Those maxima/minima at CMLs of $20 / 180^{\circ}$ and $200 / 0^{\circ}$ are associated with the attenuation emission coming, respectively, from the southern and northern hemispheres. The corresponding electron densities are found to be $\sim 7 \times 10^{4} \mathrm{~cm}^{-3}$ at the maxima, and less by one order of magnitude at the minima. Those values provide the average variation of the HOM extinction frequencies versus the central meridian longitude. However we find a noteworthy attenuation of HOM emissions between 5 and $3 \mathrm{MHz}$, respectively, when the CML range varies from 180 to $270^{\circ}$. This particular feature is only observed in the case of the northern hemisphere, and close to the inclination of the northern magnetic field axis.

\subsection{Afternoon sector}

The close approach to the planet occurs in this interval on 30 December 2000 around 16:00 LT and at a distance of $137 R_{\mathrm{J}}$. The LT interval varies between 13:30 and 19:00 LT starting from 21 December 2000 and ending on
9 January 2001. On both dates, the distance to the planet was about $185 R_{\mathrm{J}}$. The points in the middle panel of Fig. 3 have been recorded during the afternoon sector, and they correspond to about $13 \%$ of the total measured points. The HOM intensity extinction principally concerns the radiation emitted from the northern hemisphere, and much less from the southern hemisphere. The intensity attenuation is only observed in the CML interval between 50 and $340^{\circ}$. Minimum and maximum frequencies were recorded at 180 and $200^{\circ}$ CML, and may be associated, respectively, with the northern and southern hemispheres. The electron density of the Io plasma torus in the afternoon sector is estimated at about $1.5 \times 10^{5} \mathrm{~cm}^{-3}$, a factor of 2 higher than in the previous sectors (late morning and early afternoon).

\subsection{Evening sector}

In the period from 10 January to 31 March 2001, the local time of the Cassini spacecraft varies between 19:30 and 21:30 LT. The distance to Jupiter increases from about $200 R_{\mathrm{J}}$ to more than $1100 R_{\mathrm{J}}$. Nearly $40 \%$ of the total amount of points has been recorded in the evening sector. In the bottom panel of Fig. 3 we show the frequency maxima of the HOM intensity attenuation observed when the central meridian longitudes are equal to about 20 and $180^{\circ}$. Those two longitudes correspond to the moment where the magnetic axis of the planet is oriented towards the observer, i.e., the Cassini spacecraft, and corresponding respectively to the longitudes of the southern and northern magnetic axes. We note that the attenuation frequency "cutoff" at around $3 \mathrm{MHz}$, as observed in the late morning and early afternoon sectors, is quasi-absent in this LT interval. The maximum frequencies of the HOM extinctions coming from the southern and northern hemispheres are, on average, on the order of 3.0 and 2.5 MHz, as shown in Fig. 3 (bottom panel). Sometimes the attenuation may reach a frequency as high as $8 \mathrm{MHz}$, particularly close to the orientation axis of the magnetic field in the northern hemisphere. The electron densities at the origin of the HOM extinctions in the southern and in the northern hemispheres are, on average, on the order of $11.0 \times 10^{4}$ and $8.0 \times 10^{4} \mathrm{~cm}^{-3}$.

\subsection{Averaged electron density in the Io plasma torus}

We summarize in Table 1 the main outcomes of our analysis. We indicate in the columns of Table 1: (a) the central meridian longitude (CML), (b) the fitted frequencies for the northern and southern hemispheres (second and third columns), (c) the electron density of the Io plasma torus (fourth and fifth columns), (d) the local time intervals and (e) the corresponding figure. The value of the electron density seems to be overall in the order of $\sim 10^{4} \mathrm{~cm}^{-3}$. The extinction of the Jovian hectometric emissions occurs in the frequency range between $470 \mathrm{kHz}$ and up $3.5 \mathrm{MHz}$. However we note, first, a particular enhancement close to the position of the 
Table 1. Estimation of the electron density in the Io plasma torus during three local time intervals: 10:40-13:00 LT (i.e., LT1), 13:30-19:00 LT (i.e., LT2), and 19:30-21:30 LT (i.e., LT3). For each hemisphere (north or south depending on the source location of the Jovian hectometric emissions) we give the variation of CML versus the attenuation band frequency, and the corresponding electron density in the Io plasma torus that is at the origin of the HOM extinction.

\begin{tabular}{|c|c|c|c|c|c|c|}
\hline & $\begin{array}{l}\text { Freq. } \\
\mathrm{MHz}\end{array}$ & $\begin{array}{l}\text { Freq. } \\
\mathrm{MHz}\end{array}$ & $\begin{array}{r}N_{e} \\
10^{3} \mathrm{~cm}^{-3}\end{array}$ & $\begin{array}{r}N_{e} \\
10^{3} \mathrm{~cm}^{-3}\end{array}$ & & \\
\hline $\begin{array}{r}\text { CML } \\
\text { degrees }\end{array}$ & $\begin{array}{r}\text { Northern } \\
\text { Hemisphere }\end{array}$ & $\begin{array}{r}\text { Southern } \\
\text { Hemisphere }\end{array}$ & $\begin{array}{r}\text { Northern } \\
\text { Hemisphere }\end{array}$ & $\begin{array}{r}\text { Southern } \\
\text { Hemisphere }\end{array}$ & LT & Figure 3 \\
\hline 0 & 0.85 & 2.37 & 8.9 & 69.2 & $10: 40-13: 00$ & top panel \\
\hline 60 & 1.23 & 2.21 & 18.7 & 60.6 & & \\
\hline 120 & 2.00 & 1.40 & 49.0 & 24.0 & & \\
\hline 180 & 2.40 & 0.72 & 69.5 & 6.50 & & \\
\hline 240 & 2.00 & 0.90 & 49.0 & 9.5 & & \\
\hline 300 & 1.23 & 1.70 & 18.7 & 35.6 & & \\
\hline 0 & 0.95 & 2.30 & 11.4 & 66.4 & 13:30-19:00 & middle panel \\
\hline 60 & 1.61 & 2.15 & 32.0 & 57.3 & & \\
\hline 120 & 2.90 & 1.26 & 104.0 & 19.6 & & \\
\hline 180 & 3.54 & 0.53 & 155.3 & 3.45 & & \\
\hline 240 & 2.90 & 0.70 & 104.0 & 6.0 & & \\
\hline 300 & 1.60 & 1.60 & 32.0 & 31.2 & & \\
\hline 0 & 0.66 & 2.95 & 5.4 & 108.0 & $19: 30-21: 30$ & bottom panel \\
\hline 60 & 1.13 & 2.76 & 16.0 & 94.2 & & \\
\hline 120 & 2.10 & 1.70 & 53.7 & 36.0 & & \\
\hline 180 & 2.56 & 0.85 & 81.0 & 9.0 & & \\
\hline 240 & 2.10 & 1.05 & 53.7 & 13.45 & & \\
\hline 300 & 1.15 & 2.10 & 16.0 & 54.30 & & \\
\hline
\end{tabular}

magnetic field axis in the northern and southern hemispheres. Secondly, the local effect is clearly seen when combining the day- $(\sim 12: 00 \mathrm{LT})$ and nightside $(\sim 20$ :00 LT) sectors, in particular for the southern hemisphere. Hence, we find that the extinction in the HOM intensity occurs at frequencies lower than $3 \mathrm{MHz}$ on the dayside and higher in the later afternoon and evening sectors, principally. Also, the maximum frequency of the attenuation band was observed in the LT2 interval (i.e., afternoon sector) with a corresponding electron density of about $1.5 \times 10^{5} \mathrm{~cm}^{-3}$ when the central meridian longitude was about $180^{\circ}$. Beside those fitted electron densities, we find that the frequency attenuation can reach higher values of up to $8 \mathrm{MHz}$, which corresponds to a density of about $8 \times 10^{5} \mathrm{~cm}^{-3}$. This has been principally observed when the Cassini spacecraft was in the evening sector of the planet.

\section{Discussion}

We analyze the extinction of the Jovian hectometric emissions due to the Io plasma torus by assuming that the main attenuation effect of the Jovian HOM emission is related to the Io torus, as suggested by Lecacheux (1981). We show that such phenomena are regularly observed and recorded by the RPWS experiment onboard the Cassini spacecraft. The investigated period covers nearly 6 months before, during, and after the Cassini flyby of Jupiter. We derive the HOM Jovian frequencies which have been attenuated by the Io plasma torus. Those "cut-off" frequencies are found to be higher in the late afternoon and evening sectors of the planet. We estimate the corresponding electron density in the Io torus using the well-known relationship between the plasma frequency and the electron density. Hereafter we discuss our main results and reconsider the electron density measurements performed during the Voyager flyby of Jupiter, and analyze the common features between the radio and the UV observations of the Io plasma torus.

\subsection{Comparison between Cassini and Voyager observations}

It is important to compare our results with those observed by Voyager spacecraft. Hereafter we mainly refer to the dynamic spectrum as shown in Fig. 4, which is taken from Fig. 2 (top and bottom panels) of the paper of Lecacheux (1981). The total power and the polarization are displayed in the top and bottom panels, respectively. The electron density measurements were recorded, on 5 March 1979, at two time intervals, 04:00-08:30 and 14:45-16:00 SET, when the Voyager 1 orbit was above the equatorial plane. In those intervals the spacecraft was very close to Io at a distance of 


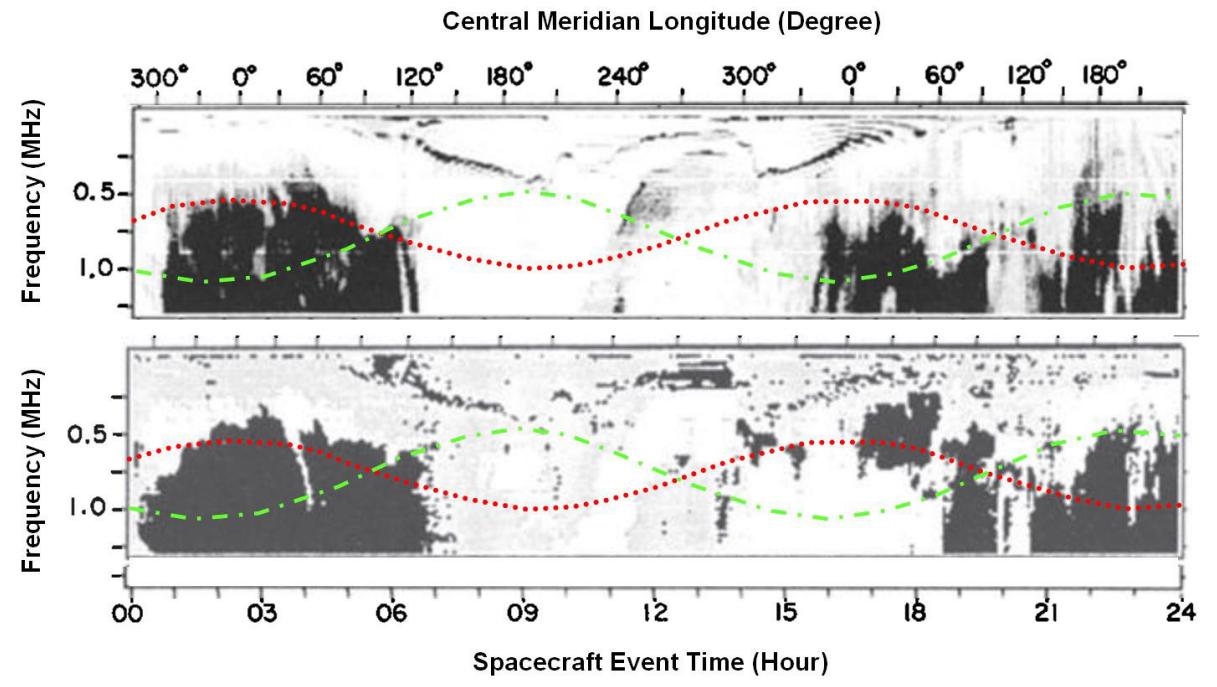

Figure 4. Daily dynamic spectrum recorded on 5 March 1979, by the Planetary Radio Experiment onboard the Voyager-1 spacecraft. The Voyager-1's closest approach was at about 12:00 spacecraft event time. The total power and the polarization are displayed in the top and bottom panels, respectively. We overlap to the Voyager dynamic spectrum two curves taken from the bottom panel of Fig. 3 . The red and the green curves indicate the variation of the HOM extinction-fitted frequencies related to the emissions coming from the northern and southern hemispheres, respectively.

approximately $5.5 R_{\mathrm{J}}$. At two occasions (i.e., 06:00-08:45 and 14:45-16:00 SET) Warwick et al. (1979) estimated from the upper hybrid frequency (below $500 \mathrm{kHz}$ ) the electron density of the Io plasma torus. Lecacheux (1981) used these electron density measurements and analyzed the influence of the Io torus on rays in the HOM frequency range. The authors found that the Io torus strongly refracts the rays below $1 \mathrm{MHz}$ around $200^{\circ} \mathrm{CML}$. Higgins et al. (1995) showed using Voyager/PRA observations that the HOM intensity extinction systematically occurred in the CML range from 120 to $300^{\circ} \mathrm{CML}$. This is in agreement with our investigations of the Cassini observations. The HOM emission attenuation principally occurred between 100 and $300^{\circ} \mathrm{CML}$, as shown in Fig. 3. This concerns the HOM radiation emitted from the northern hemisphere in particular at frequencies below $2 \mathrm{MHz}$.

In Fig. 4 we overlapped to the Voyager dynamic spectrum two curves taken from the bottom panel of Fig. 3 . The red and the green curves indicate the variation of the HOM extinction-fitted frequencies related to the emissions coming from the northern and southern hemispheres, respectively. First we note in the total power panel a weak attenuation of HOM emissions coming from the southern hemisphere at two time intervals (03:00-06:00 and 15:0020:00 SET). The extinction of the HOM radiation generated in the northern hemisphere appears between 06:00-14:00 and 21:00-24:00 SET. The Voyager right- and left-hand circular (RHC/LHC) polarizations have respectively white and black colors in the bottom panel of Fig. 4. It is interesting to note a good agreement between the Voyager HOM polarization measurements and the HOM attenuation curves.
This is the case in the time interval between 00:00 and 12:00 SET. However after this time we note a reversal in polarization when combining the Cassini HOM attenuation frequency and the Voyager polarization measurements. Hence the HOM RHC/LHC polarization of Voyager corresponds to the southern/northern HOM attenuation frequency of Cassini in the interval time 13:00-19:00/20:00-24:00 SET. This reversal of polarization in the low-frequency band of the PRA instrument has been reported by Leblanc and Daigne (1985), and interpreted as a change in the antenna-source geometry (Lecacheux and Ortega-Molina, 1987).

The combining of Voyager and Cassini observations allows us to find several similarities in the way the HOM emission is refracted by the Io plasma torus. It is possible to reconsider the estimation of the electron density in the Io torus using the method applied in our paper. The averaged attenuation frequency of about $1.2 \mathrm{MHz}$, observed at about 12:00 SET on the Voyager dynamic spectrum, corresponds to a value of about $\sim 6.0 \times 10^{4} \mathrm{~cm}^{-3}$. This is 1 order of magnitude higher than the electron density $\sim 3.5 \times 10^{3} \mathrm{~cm}^{-3}$, derived from the upper hybrid frequency.

\subsection{Comparison to visible and UV observations}

It is interesting to address the dependence of the UV observations on the central meridian longitude. Ground-based observations reported by Schneider and Trauger (1995) showed clear intensity enhancements of SII $6731 \AA$ emissions versus CMLs. These authors found that longitudes $150^{\circ}<\mathrm{CML}<210^{\circ}$ are consistently $\sim 3-4$ times brighter than at longitudes of $0^{\circ}<\mathrm{CML}<70^{\circ}$. Those two longitude 
intervals are in agreement with the maxima of attenuation frequency of the Jovian hectometric emission. The Cassini flyby of Jupiter made available the observations of the Io torus by UVIS. The visible and UV observations, from ground and space, recorded the so-called ansa region defined as the part of the torus subtended by the brightest row on the detector, plus the two neighboring rows. This UV technique lead us to the analysis of images primarily associated with the dawn and dusk sides of the torus. Steffl et al. (2006) provided temporal and azimuthal UV observations of the Io plasma torus. The authors showed that the long-term variations of torus parameters with CML, over the 45-day inbound period, are relatively small. Enhancements of the relative electron density had been observed in the interval 90-270 $\mathrm{CML}$ with maxima around $160^{\circ} \mathrm{CML}$. It is essential to note a common dependence on CML if we combine the radio HOM attenuation observations and the visible and UV ground and space recordings. The enhancement/attenuation of UV/HOM intensities occurred approximately in the same CML intervals. Hence, Schneider and Trauger (1995) and Steffl et al. (2006) reported UV intensity enhancements, respectively, in 150$210^{\circ} \mathrm{CML}$ and $90-270^{\circ} \mathrm{CML}$, which are in agreement with and comparable to the radio HOM attenuation between 80 and $290^{\circ} \mathrm{CML}$. A second interval between 0 and $70^{\circ} \mathrm{CML}$ had been reported by Schneider and Trauger (1995), which is nearly similar to the radio HOM attenuation between 300 and $80^{\circ} \mathrm{CML}$. We note the absence of this second interval in Steffl et al. (2006) for the 45-day period, from 1 October to 14 November 2000. This can be explained by the Cassini trajectory, which was above the Jovian magnetic equator at positive latitudes and indicates that principally the 45-day UV observations are associated with the intensity associated with the "northern" part of the Io plasma torus, i.e., localized above the Jovian equatorial plan.

It comes from the previous analysis that ultraviolet and hectometric wavelength observations have common features related to the "morphology" of the Io plasma torus. The UV investigations allowed analyzing of the dynamics the plasma torus at distances larger than $5.9 R_{\mathrm{J}}$, when the hectometric study allows us to remove the so-called ribbon mainly below $5.9\left(R_{\mathrm{J}}\right)$. Despite the close but different parts of the Io plasma torus, we show that enhancement/attenuation of the electron density occurs nearly in the same CML intervals, respectively, for optical and HOM observations. Those intervals are found to be in relation with the longitudes of the northern and southern hemispheres. Specifically, the maxima of enhancements/attenuations occur close to the longitudes of the tip of the magnetic dipole in the southern hemisphere $\left(20^{\circ} \mathrm{CML}\right)$ and in the northern hemisphere $\left(200^{\circ} \mathrm{CML}\right)$, respectively. This feature gives a significant indication about the role of the Jovian magnetic field as a key physical parameter in the coupling process between the planet and its satellite. The latitudinal distributions of electrons and ions appear to be principally modulated or controlled by the Jovian magnetic field.

\section{Conclusions}

We analyze in this work the intensity of extinction of the Jovian hectometric emissions as recorded by the RPWS experiment. This leads us to estimate the electron density in the Io plasma torus. We consider a period of about 6 months starting at the beginning of October 2000 and finishing at the end of March 2001. First we show that the electron density of the plasma torus is about 1 order of magnitude higher after the planet encounter. A clear local time effect takes place when the spacecraft approached the planet on the late-afternoon sector of the planet. The HOM intensity attenuation was limited to the frequency of $3 \mathrm{MHz}$. However, we note the jump of the attenuation frequency up to $4 \mathrm{MHz}$ was seen at the closest approach and later, and sometimes higher, when the Cassini spacecraft was in the late-evening sector of the Jovian magnetosphere. We find by reconsidering the Voyager observations that the electron density in the torus is one order of magnitude higher than Voyager-measured in situ observations with the upper hybrid frequency. Additionally, we show that optical and hectometric wavelength observations have common features related to the "morphology" of the Io plasma torus. The maxima of enhancements/attenuations of UV/HOM observations occur close to the longitudes of the tip of the magnetic dipole in the southern hemisphere $\left(20^{\circ}\right.$ CML) and in the northern hemisphere $\left(200^{\circ} \mathrm{CML}\right)$, respectively. This dependence is a significant indication about the importance of the Jovian magnetic field as a physical parameter in the coupling process between Jupiter and Io, in particular its effect on the latitudinal distributions of electrons and ions. Future perspectives will place emphasis on the analysis of the relationship between the radio occultation of the Jovian hectometric emissions and the motion of electrons and ions in the Io plasma torus. Future missions such as NASA's Juno (Bolton and the Juno Team, 2006) and/or ESA's JUICE (JUpiter Icy moons Explorer) (Grasset et al., 2013), planned to be launched in the next decade, can be used for the validation of our results.

Acknowledgements. The authors are grateful to D. A. Gurnett and W. S. Kurth, principal investigator and co-investigator of the RPWS experiment onboard Cassini, for making the data available for this work.

Topical Editor E. Roussos thanks two anonymous referees for their help in evaluating this paper.

\section{References}

Bagenal, F.: Empirical model of the Io plasma torus: Voyager measurements, J. Geophys. Res., 99, 11043-11062, 1994.

Bagenal, F. and Sullivan, J. D.: Direct plasma measurements in the Io torus and inner magnetosphere of Jupiter, J. Geophys. Res., 86, 8447-8466, 1981.

Bagenal, F., Crary, F. J., Stewart, A. I. F., Schneider, N. M., Gurnett, D. A., Kurth, W. S., Frank, L. A., and Paterson, W. R.: Galileo 
measurements of plasma density in the Io torus, Geophys. Res. Lett., 24, 2119-2122, 1997.

Barrow, C. H. and Desch, M. D.: Solar wind control of Jupiter's hectometric radio emission, Astron. Astrophys., 213, 495-501, 1989.

Bolton, S. and the Juno Team: The Juno Mission, in International Workshop on Instrumentation for Planetary Missions, European Planetary Science Congress, Contribution No. 1683, 2006.

Boudjada, M. Y., Galopeau, P. H. M., and Rucker, H. O.: Jovian hectometric beam observed by PWS and WAVES experiments on board Galileo and Wind spacecraft, Planet. Space Sci., 49, 1151-1158, 2001.

Boudjada, M. Y., Galopeau, P. H. M., Rucker, H. O., Lecacheux, A., Mebarki, N., Macher, W., and Voller, W.: Morphological aspects of the attenuation bands associated with Jovian hectometric radiation, J. Geophys. Res., 116, A11208, doi:10.1029/2010JA016354, 2011.

Brown, M. E. and Bouchez, A. H.: The response of Jupiter's magnetosphere to an outburst on Io, Science, 278, 268-271, 1997.

Burke, B. F. and Franklin, K. L.: Observations of a variable radio source associated with planet Jupiter, J. Geophys. Res., 60, $213-$ $217,1955$.

Esposito, L. W., Barth, C. A., Colwell, J. E., Lawrence, G. M., McClintock, W. E., Stewart, A. I. F., Keller, H. U., Korth, A., Lauche, H., Festou, M. C., Lane, A. L., Hansen, C. J., Maki, J. N., West, R. A., Jahn, H., Reulke, R., Warlich, K., Shemansky, D. E., and Yung, Y. L.: The Cassini Ultraviolet Imaging Spectrograph investigation, Space Sci. Rev., 115, 299-361, 2004.

Galopeau, P. H. M. and Boudjada, M. Y.: Solar wind control of Jovian auroral emissions, J. Geophys. Res., 110, A09221, doi:10.1029/2004JA010843, 2005.

Galopeau, P. H. M., Boudjada, M. Y., and Rucker, H. O.: Evidence of Jovian active longitude: 1. Efficiency of cyclotron maser instability, J. Geophys. Res., 109, A12217, doi:10.1029/2004JA010459, 2004.

Grasset, O., Dougherty, M. K., Coustenis, A., Bunce, E. J., Erd, C., Titov, D., Blanc, M., Coates, A., Drossart, P., Fletcher, L. N., Hussmann, H., Jaumann, R., Krupp, N., Lebreton, J.-P., Prieto-Ballesteros, O., Tortora, P., Tosi, F., and Van Hoolst, T.: JUpiter ICy moons Explorer (JUICE): An ESA mission to orbit Ganymede and to characterise the Jupiter system, Planet. Space Sci., 78, 1-21, 2013.

Green, J. L., Thieman, J. R., Higgins, C., Fung, S. F., Candey, R. M., and Aist-Sagara, L.: Lane features in Jovian hectometric radio emissions, in Planetary Radio Emissions III, edited by: Rucker, H. O., Bauer, S. J., and Kaiser, M. L., p. 91, Austrian Acad. of Sci. Press, Vienna, 1992.

Gurnett, D. A., Kurth, W. S., Roux, A., Bolton, S. J., and Kennel, C. F.: Galileo plasma wave observations in the Io plasma torus near Io, Science, 274, 391-392, 1996.

Gurnett, D. A., Kurth, W. S., Menietti, J. D., and Persoon, A. M.: An unusual rotationally modulated attenuation band in the Jovian hectometric radio emission spectrum, Geophys. Res. Lett., 25, 1841-1844, 1998.

Gurnett, D. A., Kurth, W. S., Kirchner, D. L., Hospodarsky, G. B., Averkamp, T. F., Zarka, P., Lecacheux, A., Manning, R., Roux, A., Canu, P., Cornilleau-Wehrlin, N., Galopeau, P., Meyer, A., Bostrom, R., Guastafsson, G., Wahlund, J.-E., Aahlen, L., Rucker, H. O., Ladreiter, H. P., Macher, W., Woolliscroft, L. J.
C., Alleyne, H., Kaiser, M. L., Desch, M. D., Farrell, W. M., Harvey, C. C., Louarn, P., Kellogg, P. J., Goetz, K., and Pedersen, A.: The Cassini radio and plasma wave science investigation, Space Sci. Rev., 114, 395-463, 2004.

Higgins, C. A., Green, J. L., Thieman, J. R., Fung, S. F., and Candey, R. M.: Structure within Jovian hectometric radiation, J. Geophys. Res., 100, 19487-19496, 1995.

Higgins, C. A., Thieman, J. R., Fung, S. F., Green, J. L., and Candey, R. M.: Latitudinal structure within Jovian hectometric radiation, J. Geophys. Res., 103, 26679-26686, 1998.

Kaiser, M. L.: A low frequency radio survey of the planets with RAE 2, J. Geophys. Res., 82, 1256-1260, 1977.

Krüger, H., Geissler, P., Horányi, M., Graps, A. L., Kempf, S., Srama, R., Moragas-Klostermeyer, G., Moissl, R., Johnson, T. V., and Grün, E.: Jovian dust streams: A monitor of Ioś volcanic plume activity, Geophys. Res. Lett., 30, 2101, doi:10.1029/2003GL017827, 2003.

Kupo, I., Mekler, Y., and Eviatar, A.: Detection of ionized sulfur in the jovian magnetosphere, Astrophys. J., 205, L51-L54, 1976.

Leblanc, Y. and Daigne, G.: Broadband Jovian kilometric radiation: New results on polarization and beaming, J. Geophys. Res., 90, 12073-12080, 1985.

Lecacheux, A.: Ray tracing in the Io plasma torus: Application to the PRA observations during Voyager 1 ś closest approach, J. Geophys. Res., 86, 8523-8528, 1981.

Lecacheux, A. and Ortega-Molina, A.: Polarization and localization of the Uranian radio sources, J. Geophys. Res., 92, 15148-15158, 1987.

Menietti, J. D., Gurnett, D. A., Spencer, J. R., and Stansberry, J. A.: Relationship between Jovian hectometric attenuation lanes and Io volcanic activity, Radio Sci., 36, 1723-1731, 2001.

Menietti, J. D., Gurnett, D. A., Hospodarsky, G. B., Higgins, C. A., Kurth, W. S., and Zarka, P.: Modeling radio emission attenuation lanes observed by the Galileo and Cassini spacecraft, Planet. Space Sci., 51, 533-540, 2003.

Ortega-Molina, A. and Lecacheux, A.: Polarization of Jovian hectometric emission, J. Geophys. Res., 96, 11441-11453, 1991.

Steffl, A. J., Stewart, A. I. F., and Bagenal, F.: Cassini UVIS observations of the Io plasma torus. I. Initial results, Icarus, 172, 78-90, 2004a.

Steffl, A. J., Bagenal, F., and Stewart, A. I. F.: Cassini UVIS observations of the Io plasma torus. II. Radial variations, Icarus, 172, 91-103, 2004b.

Steffl, A. J., Delamere, P. A., and Bagenal, F.: Cassini UVIS observations of the Io plasma torus. III. Modeling temporal and azimuthal variability, Icarus, 180, 124-140, doi:10.1016/j.icarus.2005.07.013, 2006.

Steffl, A. J., Delamere, P. A., and Bagenal, F.: Cassini UVIS observations of the Io plasma torus. IV. Observations of temporal and azimuthal variability, Icarus, 194, 153-165, doi:10.1016/j.icarus.2007.09.019, 2008.

Warwick, J. W., Pearce, J. B., Riddle, A. C., Alexander, J. K., Desch, M. D., Kaiser, M. L., Thieman, J. R., Carr, T. D., Gulkis, S., Boischot, A., Harvey, C. C., and Pedersen, B. M.: Voyager1 planetary radio astronomy observations near Jupiter, Science, 204, 995-998, 1979.

Wu, C. S. and Lee, L. C.: A theory of the terrestrial kilometric radiation, Astrophys. J., 230, 621-626, 1979. 